\title{
Network Analysis of Fuzzy Bi-serial and Parallel Servers with a Multistage Flow Shop Model
}

\author{
$\underline{\text { Sameer Sharma }}^{\text {a }}$, Deepak Guptab and Seema ${ }^{\mathrm{a}}$ \\ ${ }^{a}$ Department of Mathematics, D A V College, Jalandhar, Punjab, India \\ ${ }^{b}$ Department of Mathematics, M.M. university, Mullana, Haryana, India \\ Email: samsharma31@gmail.com
}

\begin{abstract}
Queuing theory is the one of the classical mathematical tool for study waiting lines or queues. In queuing theory a mathematical model is constructed to find various queue characteristics like average waiting time, mean queue length and number of the customers etc. Infact, waiting for service in queues has become an integral part of our daily life. Queues are often undesirable as they cost time, money and resources. Generally, queues come into play when the service resources are not sufficient to satisfy the demand. Most of the queuing optimization problems are static in nature in which the system characteristics are constant with respect to time or deterministic. In real life situations, one has to move from descriptive where all parameters are given to the prescriptive or normative where parameter changes with time and may be uncertain or vague in nature. In present scenario, it is difficult to find an exact distribution of arrival, service rate for the queuing applications. To express these rates mostly the linguistic expressions such as 'service is fast', 'service is slow' or 'service speed is moderate' are used. Therefore, it is more realistic to say the service rate, arrival rate etc are more possiblistic rather than probabilistic. Under these circumstances the fuzzy queuing models are more realistic approach than classical queuing theory model.

From many decades, job scheduling has been a constructive area of research for deciding the order in which the jobs are to be processed. Proper scheduling of jobs not only save time but also increase the efficiency of the system. Flow shop scheduling models are effective tools for the optimal allocation of scare resources with objective of optimizing one or several criterions.

In this paper a linkage between network of queues consisting of bi-serial and parallel servers linked to a common serve (phase I) with a multistage flow shop scheduling model (Phase II) is considered to deal with real world fuzzy network problem. Fuzzy sets are used to model uncertain arrival and service rates. The objective considered is average waiting time, mean queue length and total completion time. The triangular fuzzy membership function, $\alpha$-cut approach and various fuzzy arithmetic operations are considered to estimate the uncertainty associated with input parameters. As problem related to completion time is NP-hard, a heuristic algorithm is developed to find the solution with maximum grades for objective. The developed algorithm is tested with numerical computation. The results of proposed model is useful and significant for system designers and practitioners.
\end{abstract}

The model finds its applications in manufacturing or assembling line process in which units processed through a series of stations, each performing a given task. Practical situation can be observed in a registration process (vehicle registration) where the registrants have to visit a series of desks (advisor, department chairperson, cashier, etc.), or in a clinical physical test procedure where the patients have to pass through a series of stages (lab tests, electrocardiogram, chest X-ray etc.).

Keywords: Bi-serial servers, parallel servers, fuzzy arrival rate, service rate, mean queue length 


\section{INTRODUCTION}

In today's scenario waiting in queues is a common phenomenon that occurs in every facet of our lives. Most of queuing optimization problems is static in nature. But in many real life situations the system characteristics are described by linguistic word such as 'fast', 'slow' or 'moderate' etc. The fuzzy queue model is more realistic to handle these situations than a classical queuing theory model. In this paper a linkage model between network of queues consisting of bi-serial and parallel servers connected to a common server with a multistage flow shop scheduling model is considered. The triangular fuzzy membership function has been used to analyze the model. The total completion time of jobs/tasks in network of queues (Phase I of service) is the setup time for first machine in phase II of service consisting of ' $m$ ' machines in series. A heuristic algorithm is proposed to find the optimal sequence of jobs processing minimizing the makespan with minimum possible queue length and average waiting time of jobs in fuzzy environment.

The rest of the paper is organized as follows: Section 2 reviews the literature in context with proposed model. Section 3 deals with description of proposed fuzzy queuing scheduling linkage model. Section 4 deals with the solution methodology used to find various system characteristics. Section 5 describes the algorithm proposed for optimizing various characteristics values. Section 6 tests the efficiency of the proposed algorithm by a numerical illustration. The paper is concluded in Section 7 followed by the references.

\section{LITERATURE REVIEW}

The history of queuing theory goes back 100 years. Queuing theory has its beginning in the research work of a Danish engineer Erlang (1909), when he applied this theory extensively to study the behaviour of telephone network. It has been observed that only limited amount of literature is available on fuzzy analysis, although a significant amount of work has been done on fuzzy queues in the recent past. Buckley et al (1990) used $\alpha$ cuts to evaluate fuzzy equations. Nagoorgani and Retha (2006) studied tandem queues in fuzzy environment. Singh, T.P. et al (2005) studied the transient behaviour of a queuing network with parallel biserial queues. Kumar et al (2006) described the study state behaviour of a queue model comprised of two subsystems with bi-serial channels linked with a common channel. Robert and Ritha (2010) proposed machine interference problem with fuzzy environment. Seema et al (2013) has derived various characteristics of a queue network in which two parallel bi-serial servers are linked to a common server in series under fuzzy environment.

As per literature only some research has been made to establish a linkage between the queuing and scheduling model. Kumar et al (2007) discussed the concept of linkage of queues to a flow shop scheduling system. Gupta et al (2012) and Sharma et al (2013) studied the linkage of a parallel bi-serial queue network with a flow shop scheduling model with an effort to optimize total flow time, waiting time and service time. In this paper, the fuzzy membership functions based on Zadeh's extension principle (1978) is applied to develop a linkage network of queue model comprised of bi-serial and parallel channels linked in series to a common server with a multistage flow shop scheduling model as given by Seema et al (2014).

\section{PROPOSED MODEL}

The entire model is comprised of three servers $\mathrm{S}_{1}, \mathrm{~S}_{2}, \mathrm{~S}_{3}$. The server $\mathrm{S}_{1}$ consists of bi-serial service servers $S_{11}$ and $S_{12}$. The server $S_{2}$ contains two parallel servers $S_{21}$ and $S_{22}$. Server $S_{3}$ is commonly linked in series with each of two servers $S_{1}$ and $S_{2}$ for completion of phase service demanded either at the servers $S_{1}$ or $S_{2}$. The service time at the servers $\mathrm{S}_{\mathrm{ij}}(i, j=1,2)$ are exponentially distributed. Let mean service rate at $\mathrm{S}_{\mathrm{ij}}(i, j=1$,

2) be $\tilde{\mu}_{1}, \tilde{\mu}_{2}, \tilde{\mu}_{3}, \tilde{\mu}_{4}$ and $\tilde{\mu}_{5}$ at $S_{3}$ respectively. Queues $Q_{1}, Q_{2}, Q_{3}, Q_{4}$ and $Q_{5}$ are said to be formed in front of the servers if they are busy.

Customers coming at rate $\tilde{\lambda}_{1}$ after completion of service at $S_{11}$ will go to the network of the servers $S_{11} \rightarrow S_{12}$ or $S_{11} \rightarrow S_{3}$ with possibilities $\tilde{p_{12}}$ or $\tilde{p_{13}}$ such that $\tilde{p_{12}}+\tilde{p_{13}}=1$. The customers coming at rate $\tilde{\lambda}_{2}$ after completion of service at $S_{12}$ will go to the network of the servers $S_{12} \rightarrow S_{11}$ or $S_{12} \rightarrow S_{3}$ with possibilities $\tilde{p_{21}}$ or $\tilde{p_{23}}$ such that $\tilde{p_{21}}+\tilde{p_{23}}=1$. The customers coming at rate $\tilde{\lambda}_{3}$ after completion of service at $\mathrm{S}_{21}$ will go to the network of the server $S_{21} \rightarrow S_{3}$ and those coming at rate $\tilde{\lambda}_{4}$ after completion of service at $\mathrm{S}_{22}$ will go to the network of the server $S_{22} \rightarrow S_{3}$. The phase II of service has a system of servers (machines) 
in series. After completing the phase I of service, various jobs/tasks have to be processed through the system of machines in series. Further, the completion time (waiting time + service time) of jobs in getting phase I of service will be the setup time for first machine in phase II of service.

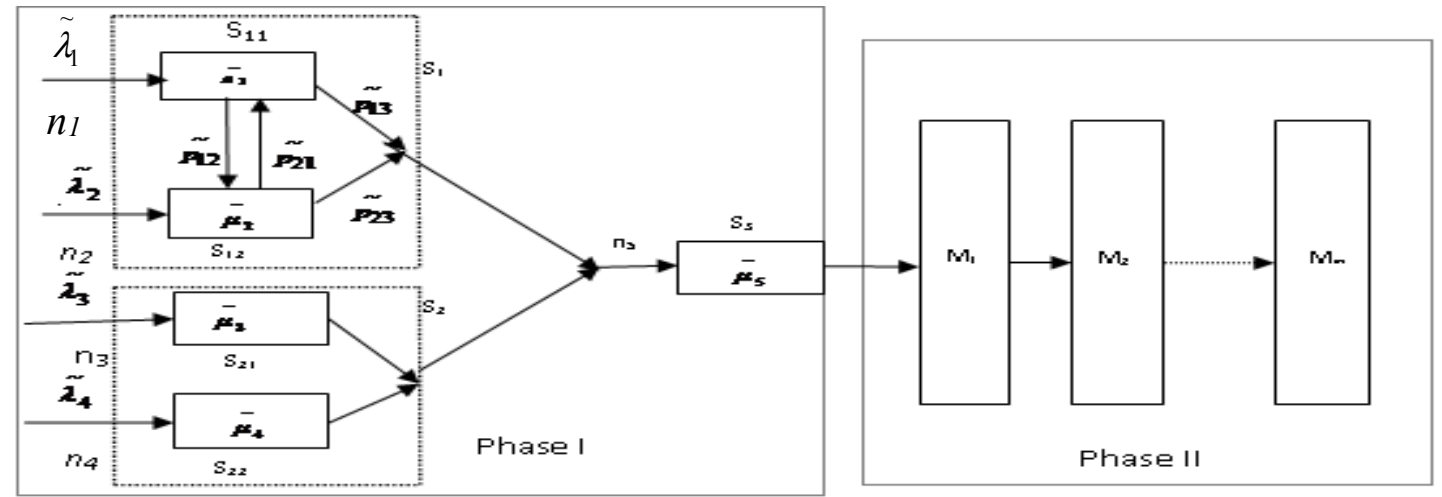

Figure 1: Fuzzy Queue Model

To estimate a parameter as a fuzzy number and to change the input data (Arrival rate, Service rate) into the fuzzy numbers, the method of Buckley and Qu method (1990) is used.

If $\tilde{A}=\left(a_{1}, a_{2}, a_{3}\right)$ be a triangular fuzzy numbers, then by using $\alpha$ - cut approach, $A^{\alpha}$ can be derived as follows: $\forall \alpha \in[0,1]$, we have $\frac{a_{1}^{(\alpha)}-a_{1}}{a_{2}-a_{1}}=\alpha ; \frac{a_{3}-a_{3}^{(\alpha)}}{a_{3}-a_{2}}=\alpha$,

We get $a_{1}^{(\alpha)}=\left(a_{2}-a_{1}\right) \alpha+a_{1} ; a_{3}^{(\alpha)}=a_{3}-\left(a_{3}-a_{1}\right) \alpha$.Thus, $A^{\alpha}=\left[a_{1}^{(\alpha)}, a_{3}^{(\alpha)}\right]=\left[\left(a_{2}-a_{1}\right) \alpha+a_{1}, a_{3}-\left(a_{3}-a_{1}\right) \alpha\right]$.

Since the arrival rate $(\tilde{\lambda})$, service rate $(\tilde{\mu})$ are not known precisely, therefore, we use triangular fuzzy numbers to represent them. Let $\tilde{\lambda}=\left(\lambda_{1}, \lambda_{2}, \lambda_{3}\right), \tilde{\mu}=\left(\mu_{1}, \mu_{2}, \mu_{3}\right)$, where $\lambda_{1}<\lambda_{2}<\lambda_{3}, \mu_{1}<\mu_{2}<\mu_{3}$ based on subjective judgment. The membership function for arrival rate $(\tilde{\lambda})$ and service rate $(\tilde{\mu})$ are defined as follows:

$\eta_{\tilde{\lambda}}(\tilde{\lambda})=\left\{\begin{array}{ll}0 & \text { if } \lambda<\lambda_{1} \\ \frac{\lambda-\lambda_{1}}{\lambda_{2}-\lambda_{1}} & \text { if } \lambda_{1} \leq \lambda \leq \lambda_{2} \\ \frac{\lambda_{3}-\lambda}{\lambda_{3}-\lambda_{2}} & \text { if } \lambda_{2} \leq \lambda \leq \lambda_{3} \\ 0 & \text { if } \lambda \geq \lambda_{3}\end{array}\right.$ and $\eta_{\tilde{\mu}}(\tilde{\mu})=\left\{\begin{array}{ll}0 & \text { if } \mu<\mu_{1} \\ \frac{\mu-\mu_{1}}{\mu_{2}-\mu_{1}} & \text { if } \mu_{1} \leq \mu \leq \mu_{2} \\ \frac{\mu_{3}-\mu}{\mu_{3}-\mu_{2}} & \text { if } \mu_{2} \leq \mu \leq \mu_{3} \\ 0 & \text { if } \mu \geq \mu_{3}\end{array}\right.$.

Let the fuzzy arrival rates are $\tilde{\lambda}_{1}, \tilde{\lambda}_{2}$; fuzzy service rates are $\tilde{\mu}_{1}, \tilde{\mu}_{2}, \tilde{\mu}_{3}$ and the various fuzzy possibilities $\tilde{p_{12}}, \tilde{p_{13}}, \tilde{p_{21}}, \tilde{p_{23}}$ are defined as follows

$\tilde{\lambda}_{i}=\left(\lambda_{i}^{1}, \lambda_{i}^{2}, \lambda_{i}^{3}\right), \tilde{\mu_{i}}=\left(\mu_{i}^{1}, \mu_{i}^{2}, \mu_{i}^{3}\right)$ and $\tilde{p_{i j}}=\left(p_{i j}^{1}, p_{i j}^{2}, p_{i j}^{3}\right)$ for various values of $i \& j$

Therefore, $\lambda_{i}^{\alpha}=\left[\alpha\left(\lambda_{i}^{2}-\lambda_{i}^{1}\right)+\lambda_{i}^{1}, \lambda_{i}^{3}-\alpha\left(\lambda_{i}^{3}-\lambda_{i}^{2}\right)\right], \mu_{i}^{\alpha}=\left[\alpha\left(\mu_{i}^{2}-\mu_{i}^{1}\right)+\mu_{i}^{1}, \mu_{i}^{3}-\alpha\left(\mu_{i}^{3}-\mu_{i}^{2}\right)\right]$ and

$$
p_{i j}^{\alpha}=\left[\alpha\left(p_{i j}^{2}-p_{i j}^{1}\right)+p_{i j}^{1}, p_{i j}^{3}-\alpha\left(p_{i j}^{3}-p_{i j}^{2}\right)\right] \text {. }
$$

In classical queuing theory, arrival rates and service times are required to follow certain probability distribution. The system characteristics of interest are the average queue length and the expected waiting time of customers / jobs. From the previous knowledge of queue theory as given by Gupta et al (2007), The average queue length and the mean expected number of customers for a bi-serial and two parallel servers linked in series with a common server are as follows: 
Sharma et al., Network analysis of fuzzy bi-serial and parallel servers....

Average queue length $=L=\frac{\rho_{1}}{1-\rho_{1}}+\frac{\rho_{2}}{1-\rho_{2}}+\frac{\rho_{3}}{1-\rho_{3}}+\frac{\rho_{4}}{1-\rho_{4}}+\frac{\rho_{5}}{1-\rho_{5}}=L_{1}+L_{2}+L_{3}+L_{4}+L_{5}$

where, $\rho_{1}=\frac{\left(\lambda_{1}+\lambda_{2} p_{21}\right)}{\mu_{1}\left(1-p_{12} p_{21}\right)}, \rho_{2}=\frac{\left(\lambda_{2}+\lambda_{1} p_{12}\right)}{\mu_{2}\left(1-p_{12} p_{21}\right)}, \rho_{3}=\frac{\lambda_{1}^{\prime}}{\mu_{1}^{\prime}}, \rho_{4}=\frac{\lambda_{2}^{\prime}}{\mu_{2}^{\prime}}$ and

$\rho_{5}=\frac{\lambda_{1}^{\prime}+\lambda_{2}^{\prime}}{\mu_{3}}+\frac{p_{13}\left(\lambda_{1}+\lambda_{2} p_{21}\right)+p_{23}\left(\lambda_{2}+\lambda_{1} p_{12}\right)}{\mu_{2}\left(1-p_{12} p_{21}\right)}$.

Also, Expected waiting time $=E(w)=\frac{L}{\lambda}$, where $\lambda=\lambda_{1}+\lambda_{2}+\lambda_{1}^{\prime}+\lambda_{2}^{\prime}$.

Since, $\rho_{1}=\frac{\lambda_{1}+\lambda_{2} p_{21}}{\mu_{1}\left(1-p_{12} p_{21}\right)}$, therefore $\rho_{1}^{\alpha}=\frac{\lambda_{1}^{\alpha}+\lambda_{2}^{\alpha} p_{21}^{\alpha}}{\mu_{1}^{\alpha}\left(1-p_{12}^{\alpha} p_{21}^{\alpha}\right)} ;$ and ${ }^{\alpha} L_{1}=\frac{{ }^{\alpha} \rho_{1}}{1-{ }^{\alpha} \rho_{1}}$.

On Substituting $\alpha=0$ and $\alpha=1$, an approximate triangular fuzzy number, $\tilde{L}_{1}$ is obtained as

$Z_{1}=\left(\frac{\frac{\lambda_{1}^{1}+\lambda_{2}^{1} p_{21}^{1}}{\left(1-p_{12}^{3} p_{21}^{3}\right) \mu_{1}^{3}}}{1-\frac{\lambda_{1}^{3}+\lambda_{2}^{3} p_{21}^{3}}{\left(1-p_{12}^{1} p_{21}^{1}\right) \mu_{1}^{1}}}, \frac{\frac{\lambda_{1}^{2}+\lambda_{2}^{2} p_{21}^{2}}{\left(1-p_{12}^{2} p_{21}^{2}\right) \mu_{1}^{2}}}{1-\frac{\lambda_{1}^{2}+\lambda_{2}^{2} p_{21}^{2}}{\left(1-p_{12}^{2} p_{21}^{2}\right) \mu_{1}^{2}}}, \frac{\frac{\lambda_{1}^{3}+\lambda_{2}^{3} p_{21}^{3}}{\left(1-p_{12}^{1} p_{21}^{1}\right) \mu_{1}^{1}}}{1-\frac{\lambda_{1}^{1}+\lambda_{2}^{1} p_{21}^{1}}{\left(1-p_{12}^{3} p_{21}^{3}\right) \mu_{1}^{3}}}\right)=\left(L_{1}^{1}, L_{1}^{2}, L_{1}^{3}\right)$; where

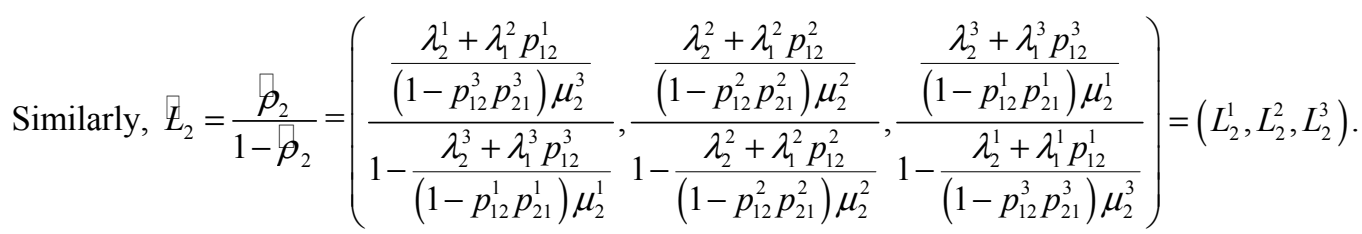

Similarly, ${ }^{\alpha} L_{3}=\frac{{ }^{\alpha} \rho_{3}}{1-^{\alpha} \rho_{3}}=\left[\frac{\frac{\alpha\left(\lambda_{11}^{2}-\lambda_{11}^{1}\right)+\lambda_{11}^{1}}{\mu_{11}^{3}-\alpha\left(\mu_{11}^{3}-\mu_{11}^{2}\right)}}{1-\frac{\lambda_{11}^{3}-\alpha\left(\lambda_{11}^{3}-\lambda_{11}^{2}\right)}{\alpha\left(\mu_{11}^{2}-\mu_{11}^{1}\right)+\mu_{11}^{1}}}, \frac{\frac{\lambda_{11}^{3}-\alpha\left(\lambda_{11}^{3}-\lambda_{11}^{2}\right)}{\alpha\left(\mu_{11}^{2}-\mu_{11}^{1}\right)+\mu_{11}^{1}}}{1-\frac{\alpha\left(\lambda_{11}^{2}-\lambda_{11}^{1}\right)+\lambda_{11}^{1}}{\mu_{11}^{3}-\alpha\left(\mu_{11}^{3}-\mu_{11}^{2}\right)}}\right]$

Substituting $\alpha=0$ and $\alpha=1$, an approximate triangular fuzzy number, $\tilde{L}_{3}$ is obtained as follows:

$Z_{3}=\left[\frac{\frac{\lambda_{11}^{1}}{\mu_{11}^{3}}}{1-\frac{\lambda_{11}^{3}}{\mu_{11}^{1}}}, \frac{\frac{\lambda_{11}^{2}}{\mu_{11}^{2}}}{1-\frac{\lambda_{11}^{2}}{\mu_{11}^{2}}}, \frac{\frac{\lambda_{11}^{3}}{\mu_{11}^{2}}}{1-\frac{\lambda_{11}^{1}}{\mu_{11}^{3}}}\right]=\left(L_{3}^{1}, L_{3}^{2}, L_{3}^{3}\right)$. Similarly, $Z_{4}=\left[\frac{\frac{\lambda_{21}^{1}}{\mu_{21}^{3}}}{1-\frac{\lambda_{21}^{3}}{\mu_{21}^{1}}}, \frac{\frac{\lambda_{21}^{2}}{\mu_{21}^{2}}}{1-\frac{\lambda_{21}^{2}}{\mu_{21}^{2}}}, \frac{\frac{\lambda_{21}^{3}}{\mu_{21}^{2}}}{1-\frac{\lambda_{21}^{1}}{\mu_{21}^{3}}}\right]=\left(L_{4}^{1}, L_{4}^{2}, L_{4}^{3}\right)$

Also, ${ }^{\alpha} \rho_{5}=\frac{\alpha \lambda_{1}^{\prime}+{ }^{\alpha} \lambda_{2}^{\prime}}{{ }^{\alpha} \mu_{3}}+\frac{{ }^{\alpha} p_{13}\left({ }^{\alpha} \lambda_{1}+{ }^{\alpha} \lambda_{2}^{\alpha} p_{21}\right)+{ }^{\alpha} p_{23}\left({ }^{\alpha} \lambda_{2}+{ }^{\alpha} \lambda_{1}^{\alpha} p_{12}\right)}{{ }^{\alpha} \mu_{2}\left(1-{ }^{\alpha} p_{12}{ }^{\alpha} p_{21}\right)}$. Therefore, ${ }^{\alpha} L_{5}=\frac{{ }^{\alpha} \rho_{5}}{1-^{\alpha} \rho_{5}}$.

On substituting $\alpha=0$ and $\alpha=1$, an approximate triangular fuzzy number, $\tilde{L}_{5}$ is given as $=\left(L_{5}^{1}, L_{5}^{2}, L_{5}^{3}\right)$

Therefore, Average (mean) fuzzy queue length $\tilde{L}=\tilde{L}_{1}+\tilde{L}_{2}+\tilde{L}_{3}+\tilde{L}_{4}+\tilde{L}_{5}=\left(l_{1}, l_{2}, l_{3}\right)$

The fuzzy average waiting time of the customers / jobs is $E(\tilde{W})=\frac{\tilde{L}}{\tilde{\lambda}}=\frac{\left(l_{1}, l_{2}, l_{3}\right)}{\left(\delta_{1}, \delta_{2}, \delta_{3}\right)}=\left(\frac{l_{1}}{\delta_{3}}, \frac{l_{2}}{\delta_{2}}, \frac{l_{3}}{\delta_{1}}\right)=\left(w_{1}, w_{2}, w_{3}\right)$

Also, the total fuzzy completion time $(c)$ of jobs/tasks for getting the phase I of service, i.e. when processed through a network of bi-serial and parallel queues connected to a common server is given by the formula: 
Sharma et al., Network analysis of fuzzy bi-serial and parallel servers....

$$
\tilde{C}=E(\tilde{W})+\frac{1}{\tilde{\mu}_{1} \tilde{p}_{12}+\tilde{\mu}_{1} \tilde{p}_{13}+\tilde{\mu}_{2} \tilde{p}_{21}+\tilde{\mu}_{2} \tilde{p}_{23}+\tilde{\mu}_{3}+\tilde{\mu}_{4}+\tilde{\mu}_{5}}=\left(c_{1}, c_{2}, c_{3}\right) .
$$

Further, the total fuzzy completion time $(\tilde{c})$ will be the setup time for first machine in Phase II of service. To Find the optimal sequence of jobs/tasks minimizes the total elapsed time when a set of ' $n$ ' jobs after completing the phase I of the service entered into the phase II of service consisting of ' $m$ ' machines in series with no passing and pre-emission is allowed the following result as given in Sharma et al (2013) is used:

Statement: Let $n$ jobs $J_{1}, J_{2}, J_{3} \ldots J_{n}$ are processed through ' $m$ ' machines $\mathrm{M}_{\mathrm{j}}(j=1,2 \ldots m)$ in order $\mathrm{M}_{1}-\mathrm{M}_{2}$ $\mathrm{M}_{3}-\ldots \ldots-\mathrm{M}_{\mathrm{m}}$ with no passing allowed. Let $A_{i, j}$ represents the crisp values of fuzzy processing time $\tilde{A}_{i, j}$ of $i^{t h}$ job $(i=1,2, \ldots, n)$ on $j^{\text {th }}$ machine $(j=1,2, \ldots, m)$ such that $\min A_{i, s} \geq \max A_{i,(s+1)} ; s=1,2, \ldots,(m-2)$, then the optimal schedule minimizing the total elapsed time is given by the following decision rule:

Job $J_{k}$ proceeds job $J_{k+1}$ if $\min \left\{G_{k}, H_{k+1}\right\}<\min \left\{G_{k+1}, H_{k}\right\}$; where $G_{i}=A_{i, 1}+A_{i, 2}+---+A_{i,(m-1)}$ and $H_{i}=A_{i, 2}+A_{i, 3}+---+A_{i, m}$.

Since, the system characteristics are described by membership function; it preserves the fuzziness of input information. However, the designer would prefer one crisp value for the system characteristics rather than fuzzy set. In order to overcome this problem we defuzzify the fuzzy values of system characteristic by using the Robust Ranking Technique. Robust Ranking technique (2011) which satisfies compensation, linearity and additive properties, provide results which are consistent with human.

If $\tilde{A}=\left(a_{1}, a_{2}, a_{3}\right)$ be a triangular fuzzy number, then robust ranking for the fuzzy number $\tilde{A}$ is defined by

$R(\tilde{A})=\int_{0}^{1}(0.5)\left(a_{\alpha}^{L}, a_{\alpha}^{U}\right) d \alpha$, where $\left(a_{\alpha}^{L}, a_{\alpha}^{U}\right)=\left\{\left(a_{2}-a_{1}\right) \alpha+a_{1}, a_{3}-\left(a_{3}-a_{2}\right) \alpha\right\}$ is the $\alpha$ - cut for the fuzzy number $\tilde{A}=\left(a_{1}, a_{2}, a_{3}\right)$.

\section{ALGORITHM}

The following algorithm gives the procedure to determine the optimal sequence of jobs/tasks for the linkage network of bi-serial and parallel queues coupled to a common server with a multistage flow shop setup having ' $m$ ' machines in series under fuzzy environment.

Step 1: Find the mean queue length: $\tilde{L}=\tilde{L}_{1}+\tilde{L}_{2}+\tilde{L}_{3}+\tilde{L}_{4}+\tilde{L}_{5}=\left(l_{1}, l_{2}, l_{3}\right)$.

Step 2: Find the average fuzzy waiting time of the customers on the lines of Little's (1965) using relation $E(\tilde{w})=\frac{\tilde{L}}{\tilde{\lambda}}=\left(w_{1}, w_{2}, w_{3}\right)$.

Step 3: Find the total fuzzy completion time $(\tilde{c})$ of jobs/customers coming out of Phase I of the service.

Step 4: The total fuzzy completion time $(\tilde{c})$ will be the setup time for first machine in Phase II of the service.

Define the machines $\mathrm{M}_{1}, \mathrm{M}_{2} \ldots, \mathrm{M}_{\mathrm{m}}$ with processing time $\tilde{A_{i, 1}^{\prime}}=\tilde{A_{i, 1}}+C$ and $\tilde{A_{i, 2}}, \tilde{A_{i, 3}}, \ldots, \tilde{A_{i, m}}$.

Step 5: Check the consistency of the structural conditions so as to reduce ' $n$ ' jobs ' $m$ ' machines problem to ' $n$ ' jobs 2 machine problem. If condition hold: Introduce the two fictitious machines $G$ and $H$ with processing time $G_{i}$ and $\quad H_{i}$ defined $G_{i}=\tilde{A_{i, 1}}+\tilde{A_{i, 2}}+------+\tilde{A}_{i,(m-1)}^{\sim}$ and $H_{i}=\tilde{A}_{i, 2}+\tilde{A}_{i, 3}+------+\tilde{A}_{i, m}$.

Step 6: Apply modified technique Sharma et al (2013) to find the optimal sequence(s) of jobs processing with minimum total elapsed time.

Step 7: Prepare In-out tables for the sequence of jobs as obtained in step 6. The sequence $S$ having minimum total elapsed time will be the optimal sequence for the given linkage model with mean queue

length $\tilde{L}$ and $E(\tilde{w})$ as average waiting time of jobs/tasks in queuing- scheduling linkage model. 


\section{NUMERICAL ILLUSTARTION}

The following numerical illustration is carried out to test the efficiency of the algorithm proposed:

Consider seven jobs/tasks are processed through the network of queues $Q_{1}, Q_{2}$ and $Q_{3}$ with the servers $\mathrm{S}_{1}, \mathrm{~S}_{2}$ and $S^{\prime}$. The servers $\mathrm{S}_{1}$ and $\mathrm{S}_{2}$ are parallel bi-serial servers and server $S^{\prime}$ linked in series with each of two servers $S_{1}$ and $S_{2}$. The number of the customers, fuzzy average arrival rate, fuzzy mean service rate and various associated possibilities are as given in table 1.

Table 1: Input variables for the proposed model

\begin{tabular}{|c|c|l|l|}
\hline & Arrival Rate & Service Rate & Possibilities \\
\hline$n_{1}=2$ & $\tilde{\lambda}_{1}=(2,3,4)$ & $\tilde{\mu}_{1}=(10,11,12)$ & $\tilde{p}_{12}=(0.4,0.6,0.7)$ \\
\hline$n_{2}=1$ & $\tilde{\lambda}_{2}=(5,6,7)$ & $\tilde{\mu}_{2}=(12,13,17)$ & $\tilde{p}_{13}=(0.4,0.3,0.2)$ \\
\hline$n_{3}=2$ & $\tilde{\lambda}_{3}=(3,4,5)$ & $\tilde{\mu}_{3}=(12,14,16)$ & $\tilde{p}_{21}=(0.3,0.5,0.6)$ \\
\hline$n_{4}=2$ & $\tilde{\lambda}_{4}=(2,4,5)$ & $\tilde{\mu}_{4}=(6,7,8)$ & $\tilde{p}_{23}=(0.7,0.5,0.4)$ \\
\hline$n_{5}=7$ & & $\tilde{\mu}_{5}=(12,15,16)$ & \\
\hline
\end{tabular}

The aim is to find the optimal sequence of jobs processing with minimum makespan by considering the phase I of service into account. On applying various steps of the algorithm proposed, we have

$\tilde{L}_{1}=(1.6390,3.5289,1.5471) ; \tilde{L}_{2}=(8.7381,6.0021,2.5) ; \tilde{L}_{3}=(0.3214,0.399,0.3809)$;

$\tilde{L}_{4}=(1.4997,2.0003,0.9523)$ and $\tilde{L_{5}}=(13.8094,19.8768,34.226)$

Therefore, mean queue length of customers/jobs is $\tilde{L}=\left(l_{1}, l_{2}, l_{3}\right)=(26.0076,31.808,39.6063)$.

and, the expected waiting time of the customers is $E(\tilde{w})=(1.238,1.8710,3.3005)$.

Now, on using the Robust's Ranking Techniques (2011), the crisp value for mean queue length of customers $R(\tilde{L})=32.80695$ units and that for expected waiting time is $R(\tilde{w})=2.26945$ units.

From figure 2, we observe that the mean queue length of customers/jobs $R(\tilde{L})$ in the system falls between 26.0076 and 39.6063. The most possible mean queue length of customers (jobs) is 31.808 units. Figure 3 indicates that the support of $R(\tilde{w})$ ranges from 1.2384 to 3.3005 and the most possible value for the expected waiting time in the system is 1.8710 units.

Also, the total fuzzy completion time of jobs/tasks in getting phase I of service is $\tilde{C}=(1.25249,1.88798,3.3205)$.

After being served in phase I, the various jobs/tasks entered in phase II of service having a system of machines/servers in series. The fuzzy processing times of various jobs on these machines are as follows:

Table 2: Jobs with fuzzy processing time

\begin{tabular}{|c|c|c|c|c|c|c|c|}
\hline Jobs $(i)$ & 1 & 2 & 3 & 4 & 5 & 6 & 7 \\
\hline Machine $M_{l}\left(\tilde{A}_{i, 1}\right)$ & $(7,8,9)$ & $(12,13,14)$ & $(8,10,12)$ & $(10,11,12)$ & $(9,10,11)$ & $(8,10,12)$ & $(12,13,14)$ \\
\hline Machine $M_{2}\left(\tilde{A}_{i, 2}\right)$ & $(6,7,8)$ & $(5,6,7)$ & $(4,5,6)$ & $(5,6,7)$ & $(6,7,8)$ & $(4,5,6)$ & $(5,6,7)$ \\
\hline Machine $M_{3}\left(\tilde{A}_{i, 3}\right)$ & $(3,4,5)$ & $(4,5,6)$ & $(6,7,8)$ & $(11,12,13)$ & $(8,9,10)$ & $(6,7,8)$ & $(4,5,6)$ \\
\hline
\end{tabular}




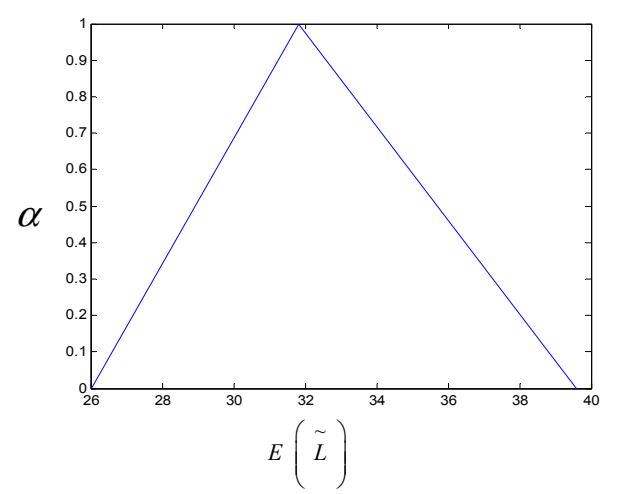

Figure 2: Expected fuzzy mean queue length

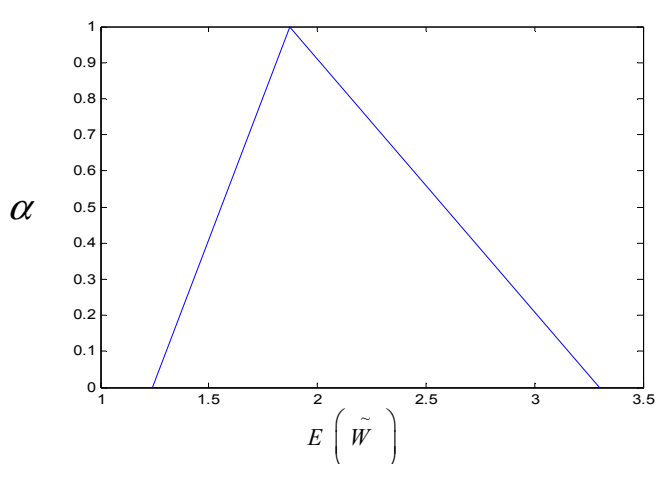

Figure 3: Expected fuzzy mean queue length

The total fuzzy completion time $(\tilde{c})$ will be the setup time of jobs on first machine in Phase II of service.

On following various steps of the algorithm proposed, the optimal sequence of jobs processing is $\mathrm{S}=4-5-$ $3-6-7-1-2$ and the minimum total elapsed time of the jobs/tasks is 100.571977 units.

\section{DISCUSSION AND CONCLUSIONS}

The fuzzy systems are very useful in two general contexts: one in situations involving highly complex system whose behaviours are not well understood and secondly, in situations where an approximate but fast solution is warranted. In this paper the fuzzy logic approach is used to represents the uncertainty or vagueness or complexity or impreciseness in the arrival rate, service rate, various possibilities and the processing time of jobs/tasks. An algorithm is developed to find the mean queue length, average waiting time and minimum possible elapsed time for the proposed queuing-scheduling linkage model in fuzzy environment. The study may further be extended by introducing more complex network of queues in phase I of service in fuzzy environment and also, by introducing some scheduling constraints like independent setup time, transportation time etc. in phase II of service in the proposed linkage model.

\section{REFERENCES}

Buckley, J. J., and Qu, Y. 1990. On using $\alpha$ cuts to evaluate fuzzy equations. Fuzzy Sets and Systems, 38, 309-312.

Gupta, D., Singh, T.P. and Kumar, R. 2007. Analysis of a network queue model comprised of biserial and parallel channels linked to a common server, Ultra Science, 19(2)M, 407-418.

Gupta, D., Sharma, S. and Sharma, S. 2012. On linkage of a flowshop scheduling model including job block criteria with a parallel biserial queue network, Computer Engineering and Intelligent System, 3(2), 17-28.

Kumar, V., Singh, T. P. , and Kumar, R. 2006. Steady state behaviour of a queue model comprised of two subsystems with biserial channels linked with a common channel. Ref Des Era JSM, 1(2), 135-152.

Little John, D. C. 1965. A proof of queuing formula: . Operation Research, 13, 400-412.

Nagoorgani, A. , and Retha, W. 2006. Fuzzy tandem queues. Acta Cienicia Indica. XXXII(M), 257-263.

Robert, L. , and Ritha, W. 2010. Machine interference problem with fuzzy environment. I. J. Contemporary Math. Science, 5(39), 1905-1912.

Seema, Gupta, D. and Sharma, S. 2013. Analysis of biserial servers linked to a common server in fuzzy environment, International Journal of Computer Applications, 68(6), 26-32.

Seema, Gupta, D. and Sharma, S. 2014. Analysis of linkage network of queues with a multistage flow shop scheduling system, International Journal of Operational Research, 21(4), 409-432.

Singh, T. P., Kumar, V., and Kumar, R. 2005. On transient behaviour of queuing network with parallel biserial queues. JMASS, 1(2), 68-75.

Yan, D. and Ng, W. 2011. Robust ranking of uncertain data, Lecture notes in Computer Science, 6587(2011), 254-268.

Zadeh, L. A. 1978. Fuzzy sets as a basis for a theory of possibility. Fuzzy Sets and Systems, 1, 3-28. 